\title{
AK-means: an automatic clustering algorithm based on K-means
}

\author{
Omar Kettani*, Faical Ramdani, Benaissa Tadili \\ Mohamed V-University, Scientific Institute, Physics of the Earth Laboratory Rabat, Morocco \\ *Corresponding author E-mail: kettani.o@gmail.com
}

Copyright $\odot 2015$ O. Kettani et al. This is an open access article distributed under the Creative Commons Attribution License, which permits unrestricted use, distribution, and reproduction in any medium, provided the original work is properly cited.

\begin{abstract}
In data mining, K-means is a simple and fast algorithm for solving clustering problems, but it requires that the user provides in advance the exact number of clusters $(\mathrm{k})$, which is often not obvious. Thus, this paper intends to overcome this problem by proposing a parameter-free algorithm for automatic clustering. It is based on successive adequate restarting of K-means algorithm. Experiments conducted on several standard data sets demonstrate that the proposed approach is effective and outperforms the related well known algorithm G-means, in terms of clustering accuracy and estimation of the correct number of clusters.
\end{abstract}

Keywords: Automatic Clustering; G-Means; K-Means; Parameter-Free Clustering

\section{Introduction}

Clustering is the process of grouping data into disjoint set called clusters such as that similarities among data members within the same cluster are maximal while similarities among data members from different clusters are minimal. The optimization of this criterion is an NP hard problem in general Euclidean space d, even when the clustering process deals with only two clusters [1]. To tackle this problem, many approximation algorithms have been proposed, aiming to find near optimal clustering solution in reasonable computational time. Most of the existing clustering algorithms depend on one or more tuning parameters, which are often difficult to determine, because they may require many empirical error-trials steps without a reliable effective result. K-means [2], the most prominent clustering algorithm has a major drawback: the user must specify the correct number of clusters in advance, which is often a difficult task when the distribution of the given data set is unknown.

In this paper, an alternative parameter free method for automatic clustering, called AK-means, is proposed. It is based on successive adequate restarting of K-means. Algorithm validation and comparative study with G-means [3], a related well known algorithm, are conducted using several real-worlds and artificial clustering data sets from the UCI Machine Learning Repository [4].

In the next section, some related works are briefly discussed. Then the proposed approach is described in Section 3. Section 4 presents application's results of this clustering method to different standard data sets and reports its performance. Finally, conclusions of the paper are summarized in Section 5.

\section{Related work}

Despite the fact that obtaining an optimal number of clusters k for a given data set is an NP-hard problem [5], several methods have been developed to find $\mathrm{k}$ automatically.

Pelleg and Moore [6] introduced the X-means algorithm, which proceed by learning k with k-means using the Bayesian Information Criterion (BIC) to score each model, and choose the model with the highest BIC score. However, this method tends to over fit when it deals with data that arise from non-spherical clusters. Tibshirani et al. [7] proposed the 
Gap statistic, which compares the likelihood of a learned model with the distribution of the likelihood of models trained on data drawn from a null distribution. This method is suitable for finding a small number of clusters, but has difficulty when $\mathrm{k}$ increases. Hamerly and Elkan [3] proposed the G-means algorithm, based on K-means algorithm, which uses projection and a statistical test for the hypothesis that the data in a cluster come from a Gaussian distribution. This algorithm works correctly if clusters are well-separated, and fail when clusters overlap and look non-Gaussian. In our experiments, G-means tends to overestimate the number of clusters, as reported in section 4.

In the present work, an alternative approach is proposed, attempting to overcome these issues.

\section{Proposed approach}

The proposed algorithm starts by setting $\mathrm{k}=$ floor $\left((\mathrm{n})^{1 / 2}\right)$; where $\mathrm{n}$ is the number of objects in the given data set. This choice is motivated by the fact that this number lies in the range from 2 to (n) ${ }^{1 / 2}$, as reported by Pal and Bezdek in [8].

Then it applies a deterministic initialization procedure proposed by the authors in [9]. K-means algorithm is applied with these initial $\mathrm{k}$ centroids, and centroid of the smallest cluster is removed, then K-means restarts with the remaining centroids. At each iteration, the maximum of $\mathrm{CH}$ cluster validity index [10] of the current partition is stored. We used this index because it is relatively inexpensive to compute, and it generally outperforms other cluster validity indices as reported by Milligan and Cooper in [11]. This process is repeated until k=2. Finally, the algorithm outputs the optimal $\mathrm{k}$ and partition corresponding to the maximum value of $\mathrm{CH}$ stored so far. This algorithm is outlined in the pseudo-code below:

Algorithm AK-means

Input: $\mathrm{D}=\left\{\mathrm{x}_{1}, \mathrm{x}_{2} \ldots \mathrm{x}_{\mathrm{n}}\right\}$ in $\mathrm{R}^{\mathrm{d}}$

Output: $k$ mutually disjoint clusters $C_{1} \ldots C_{k}$ such that $\stackrel{k}{\bigcup} C_{j}=D$

$\mathrm{j}=1$

$\mathrm{k} \leftarrow\left\lceil(\mathrm{n})^{1 / 2}\right\rceil$

$\mathrm{X} \leftarrow \mathrm{D}$

For $\mathrm{j}=1$ to $\mathrm{k}$ do

$$
\begin{aligned}
& \mathrm{C}_{\mathrm{j}} \leftarrow \operatorname{KNN\operatorname {search}}\left(\mathrm{x}_{1}, \mathrm{X},\lceil\mathrm{n} / \mathrm{k}\rceil\right) \\
& \mathrm{c}_{\mathrm{j}} \leftarrow \sum_{\mathrm{X}_{\mathrm{i}}} \in \mathrm{x}_{\mathrm{j}} /\lceil\mathrm{n} / \mathrm{k}\rceil \\
& \mathrm{X} \leftarrow \mathrm{X}-\mathrm{C}_{\mathrm{j}}
\end{aligned}
$$

End For

$[\mathrm{I}, \mathrm{c}] \leftarrow \mathrm{K}$-means $(\mathrm{D}, \mathrm{c}, \mathrm{k})$

ko $\leftarrow$ k

Io $\leftarrow$ I

$\mathrm{CHo} \leftarrow \mathrm{CH}$ (I)

While $\mathrm{k}>2$ do

$$
\begin{aligned}
& \mathrm{j} \leftarrow \operatorname{argMin}\left(\left|\mathrm{C}_{\mathrm{i}}\right|\right) \\
& \mathrm{i}<=\mathrm{k} \\
& \mathrm{c}_{\mathrm{j}} \leftarrow[] \\
& \mathrm{k} \leftarrow \mathrm{k}-1
\end{aligned}
$$


$[\mathrm{I}, \mathrm{c}] \leftarrow \mathrm{K}$-means $(\mathrm{D}, \mathrm{c}, \mathrm{k})$

if $\mathrm{CHo}<\mathrm{CH}(\mathrm{I})$ then

$$
\begin{aligned}
& \text { ko } \leftarrow \mathrm{k} \\
& \text { Io } \leftarrow \mathrm{I} \\
& \mathrm{CHo} \leftarrow \mathrm{CH}(\mathrm{I})
\end{aligned}
$$

End if

End while

Output: ko and Io

\section{Experimental results}

Algorithm validation is conducted using seven real-world clustering data sets, namely breast, iris, wine, glass, ruspini, thyroid, yeast and 14 artificial generated clustering data sets from the UCI Machine Learning Repository. Data sets s1 to s4 are generated with varying complexity in terms of spatial data distributions, which have 5000 vectors scattered around 15 predefined clusters with varying degrees of overlap. Data sets a1, a2, and a3 are generated in 2-dimensional Gaussian distribution; there are 150 vectors per cluster. Dim32 to Dim528 are high-dimensional data sets with 16 Gaussian clusters.

Silhouette index [12] which measures the cohesion based on the distance between all the points in the same cluster and the separation based on the nearest neighbor distance, was used in these experiments (bigger average silhouette value indicates a higher clustering accuracy).

In these experiments, a comparative study between G-means and AK-means is conducted on these data sets using Matlab software on a computer with Intel Core 2Duo CPU 2.8 GHZ and RAM 4.0GB memory. The number of clusters found by both algorithms, the average of silhouette values and CPU running time are reported in table 1.

In our experiments, we used $\alpha=0.0001$ the significance level of the test, for G-means script.

The results of the experiments with different data sets indicate that the proposed approach estimates the correct number of clusters, in 18 cases among 21 tested data sets. A Matlab code of the proposed approach is given in the appendix.

\section{Conclusion}

In this work, an algorithm was suggested for automatic clustering. This approach estimated the correct number of clusters in almost all tested data sets. This method was compared with the related well known algorithm, G-means, which improved for finding the correct number of clusters. The comparisons also showed that the proposed approach is better than G-means in terms of clustering accuracy.

In future work, it will be of interest to find a tighter upper bound on the number of clusters, instead of $n^{1 / 2}$, in order to reduce the number of computation's steps of the proposed approach. Another possible algorithm's speed up is to avoid unnecessary distance calculations by exploiting the triangle inequality following the method developed by Elkan in [13]. A further possible improvement of the proposed approach will consist to try more adequate similarity measures instead of Euclidean distance, in order to enhance its clustering accuracy.

\section{Acknowledgements}

This work is supported by Mohamed V University funding from P-U. Project. 
Table 1: Experimental Results of Application of G-Means and AK-Means on Different Data Sets.

\begin{tabular}{|c|c|c|c|c|c|c|c|}
\hline \multirow[b]{2}{*}{ Dataset } & \multirow[b]{2}{*}{$\mathrm{k}$} & \multicolumn{3}{|c|}{ G-means } & \multicolumn{3}{|c|}{ AK-means } \\
\hline & & $\mathrm{k}$ found & Mean Silh & CPU time (s) & $\mathrm{k}$ found & Mean Silh & CPU time (s) \\
\hline breast & 2 & 106 & 0.4226 & 5.1120 & 2 & 0.7542 & 1.0386 \\
\hline iris & 3 & 3 & 0.7786 & 2.8643 & 3 & 0.7786 & 0.3728 \\
\hline glass & 7 & 2 & 0.7879 & 0.7893 & 15 & 0.6514 & 0.5293 \\
\hline ruspini & 4 & 4 & 0.9086 & 0.9048 & 4 & 0.9086 & 0.0997 \\
\hline thyroid & 2 & 3 & 0.7773 & 0.9017 & 3 & 0.7773 & 0.4168 \\
\hline wine & 3 & 3 & 0.5043 & 0.9940 & 3 & 0.5043 & 0.3411 \\
\hline yeast & 10 & 19 & 0.2659 & 2.4021 & 2 & 0.4102 & 6.2920 \\
\hline a1 & 20 & 23 & 0.7337 & 2.0891 & 20 & 0.7892 & 5.2059 \\
\hline $\mathrm{a} 2$ & 35 & 40 & 0.7413 & 2.3931 & 35 & 0.7911 & 14.5874 \\
\hline a3 & 50 & 53 & 0.7727 & 3.7775 & 50 & 0.7949 & 27.6954 \\
\hline D31 & 31 & 31 & 0.9222 & 1.6490 & 31 & 0.9222 & 4.9055 \\
\hline $\operatorname{dim} 32$ & 16 & 111 & 0.4413 & 5.3006 & 16 & 0.9962 & 3.5238 \\
\hline $\operatorname{dim} 64$ & 16 & 109 & 0.5464 & 5.4418 & 16 & 0.9985 & 6.1691 \\
\hline $\operatorname{dim} 128$ & 16 & 111 & 0.6214 & 8.2748 & 16 & 0.9991 & 13.1553 \\
\hline $\operatorname{dim} 256$ & 16 & 106 & 0.6032 & 9.8462 & 16 & 0.9996 & 28.2731 \\
\hline $\operatorname{dim} 528$ & 16 & 109 & 0.5999 & 20.4447 & 16 & 0.9998 & 63.4478 \\
\hline R15 & 15 & 15 & 0.9361 & 1.2827 & 15 & 0.9361 & 0.4046 \\
\hline s1 & 15 & 80 & 0.5632 & 8.4497 & 15 & 0.8803 & 12.7067 \\
\hline s2 & 15 & 87 & 0.5563 & 13.4046 & 15 & 0.8009 & 18.1031 \\
\hline s3 & 15 & 73 & 0.5393 & 14.7327 & 15 & 0.6659 & 27.3990 \\
\hline s4 & 15 & 85 & 0.5315 & 25.1755 & 15 & 0.6446 & 26.1389 \\
\hline
\end{tabular}

\section{References}

[1] Aloise, D.; Deshpande, A.; Hansen, P.; Popat, P. (2009). "NP-hardness of Euclidean sum-of-squares clustering". Machine Learning 75: 245249. http://dx.doi.org/10.1007/s10994-009-5103-0.

[2] Lloyd. S. P. (1982). "Least squares quantization in PCM". IEEE Transactions on Information Theory 28 (2): $129-137$. http://dx.doi.org/10.1109/TIT.1982.1056489.

[3] Greg Hamerly and Charles Elkan. Learning the k in k-means. In Proceedings of the seventeenth annual conference on neural information processing systems (NIPS), pages 281-288, 2003

[4] Asuncion, A. and Newman, D.J. (2007). UCI Machine Learning Repository [http://www.ics.uci.edu/ mlearn/MLRepository.html]. Irvine, CA: University of California, School of Information and Computer Science.

[5] H. Spath, Clustering Analysis Algorithms for Data Reduction and Classification of Objects, Ellis Horwood, Chichester, 1980.

[6] Dan Pelleg and Andrew Moore. X-means: Extending k-means with efficient estimation of the number of clusters. In Proceedings of the 17th International Conf. on Machine Learning, pages 727-734. Morgan Kaufmann, 2000.

[7] Robert Tibshirani, Guenther Walther, and Trevor Hastie. Estimating the number of clusters in a dataset via the Gap statistic. Journal of the Royal Statistical Society B, 63:411-423, 2001. http://dx.doi.org/10.1111/1467-9868.00293.

[8] Pal, N.R. and Bezdek, J.C. (1995) On Cluster Validity for the Fuzzy c-Means Model. IEEE Transactions on Fuzzy Systems, 3 , $370-379$. http://dx.doi.org/10.1109/91.413225.

[9] Kettani, O.; Tadili, B. and Ramdani, F. - A deterministic k-means algorithm based on nearest neighbor search. International Journal of Computer Applications (0975 - 8887), Vol. 63, No.15, February 2013. http://dx.doi.org/10.5120/10544-5541.

[10] T. Calinski and J. Harabasz. A dendrite method for cluster analysis. Communications in Statistics, 3:1-27, 1974.

[11] G. W. Milligan and M. C. Cooper. An examination of procedures for determining the number of clusters in a data set. Psychometrica, 50:159179, 1985. http://dx.doi.org/10.1007/BF02294245.

[12] L. Kaufman and P. J. Rousseeuw. Finding groups in Data: "an Introduction to Cluster Analysis". Wiley, 1990. http://dx.doi.org/10.1002/9780470316801.

[13] C. Elkan, "Using the triangle inequality to accelerate k-means", ICML 2003 Conference Proceedings, p. $147 \# 153,2003$. 


\section{Appendix}

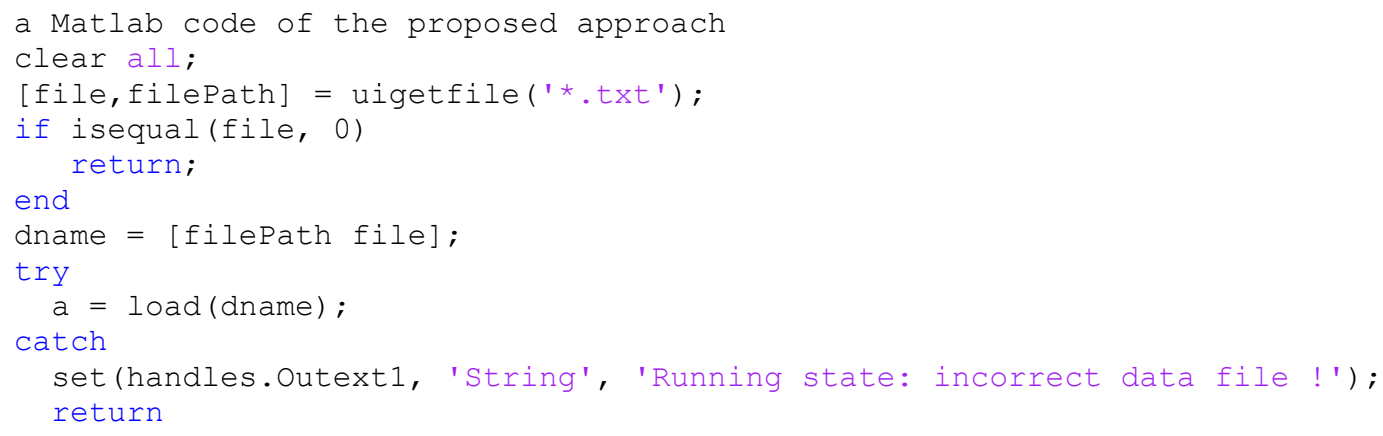

function $[\mathrm{CH}]=\mathrm{vCH}($ data, labels)

$[$ nrow, nc $]=$ size (data);

labels = double(labels);

$\mathrm{k}=\max (\mathrm{labels})$;

$[\mathrm{sw}, \mathrm{sb}]=\mathrm{V}_{-}$sumsqures (data, labels, k) ; 


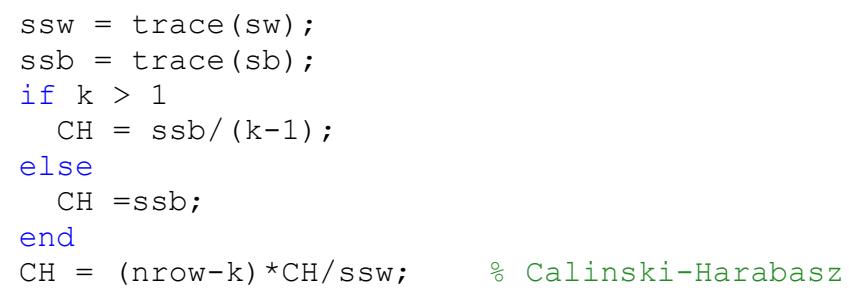

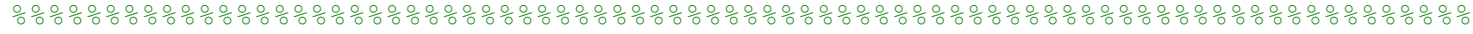

function $[\mathrm{W}, \mathrm{B}]=\mathrm{v}$ _sumsqures (data, labels, k)

if $(\operatorname{size}(l a b e l s, 1)==1)$

labels = labels';

end

$[$ ncase, $\mathrm{m}]=$ size (data);

$\mathrm{Dm}=$ mean $($ data $) ;$

$\mathrm{Dm}=$ data - Dm (ones (ncase, 1), :);

$\mathrm{T}=\mathrm{Dm}{ }^{\prime} * \mathrm{Dm}$;

$\mathrm{W}=\operatorname{zeros}(\operatorname{size}(\mathrm{T})) ;$

$\mathrm{Dm}=\operatorname{zeros}(\mathrm{k}, \mathrm{m}) ;$

for $i=1: \mathrm{k}$

if $\mathrm{k}>1$

Cindex $=$ find (labels $==i)$;

else

Cindex $=1:$ ncase;

end

$\mathrm{nk}=$ length (Cindex);

if $\mathrm{nk}>1$

datac $=\operatorname{data}($ Cindex, $:)$;

$\mathrm{m}=\operatorname{mean}($ datac $)$;

$\operatorname{Dm}(i,:)=m$;

dataC $=$ dataC $-\operatorname{repmat}(\mathrm{m}, \mathrm{nk}, 1)$;

$\mathrm{W}=\mathrm{W}+$ dataC'*dataC;

datac $=\operatorname{sum}($ datac.^2,2);

end

end

$\mathrm{B}=\mathrm{T}-\mathrm{W}$;

end

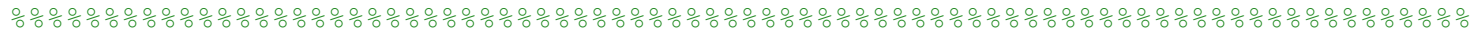

function distances $=$ calcdist2 (data, center)

$[\mathrm{n}, \mathrm{dim}]=\operatorname{size}($ data) $;$

$[$ n2, dim2] = size(center);

if $\mathrm{n} 2==1$

distances $=\operatorname{sum}\left(\right.$ data.^2, 2) $-2{ }^{*}$ data*center' + center*center';

elseif $\mathrm{n} 2==\mathrm{n}$

else

distances $=\operatorname{sum}(($ data - center $) \cdot \wedge 2,2)$;

end

error('bad number of centers');

distances $=$ distances;

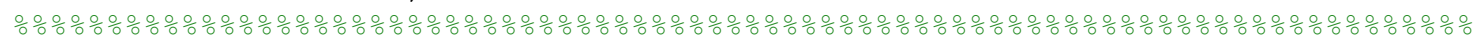

function $\mathrm{C}=$ init $(\mathrm{a}, \mathrm{k})$

$[\mathrm{n}, \mathrm{p}]=\operatorname{size}(\mathrm{a})$;

$\mathrm{Z}=\mathrm{a}$

$\mathrm{C}=[$ ]

$i \mathrm{dx}=[]$

for $j=1: k-1$

$i d x=k n n s e a r c h(z(1,:), z, r o u n d(n / k))$

$C(j,:)=\operatorname{mean}(Z(i d x,:))$

end

$\mathrm{Z}(\mathrm{idx},:)=[]$

$C(k,:)=\operatorname{mean}(\mathrm{Z}(1:$ end,$:))$ 